\title{
CLASSIFICATION OF ENVIRONMENTAL EFFECTS ON SOME EGYPTIAN COTTON GENOTYPES
}

\author{
(Received:11 .7 .2012) \\ By \\ H. A. Idris \\ Cotton Research Institute, Agriculture Research Center, Giza, Egypt.
}

\begin{abstract}
The present investigation aimed to classify environmental effects on some Egyptian cotton (Gossypium barbadense L.) genotypes. Genotypes (G.80, G90, (G83 x (G75 x 5844)) x G80 and G90 x Australian) were evaluated in six environments with respect to yield, boll components, indices and fiber properties. Environments consisted of three seasons (2009, 2010 and 2011) x two locations (Beni Souf and Minya) in Upper Egypt. A randomized complete block design with four replications was used. The results of classification of the environments revealed that the genotypes were more strongly influenced by different environments and components (locations and seasons) with respect to seed and lint yield and indices, seed index and lint percentage except G90 x Australian (seasons) was non-significant for lint percentage. G90 x Australian was the best genotype (more stable) for boll components because the variance of this genotype was non-significant under different environments and components (locations and seasons). The genotypes were not affected by locations with respect to fiber properties except G80 for fiber length and G90 x Australian for Pressely index the variance of genotypes was not significant. The present study is very important for the regional program to evaluate the genotypes.
\end{abstract}

Key words: cotton, environments, genotypes, locations, sample analysis, seasons.

\section{INTRODUCTION}

Genotype $\mathrm{x}$ environment interaction continues to be a challenging issue for plant breeders, geneticists and production agronomists who conduct crop performance trials across diverse environments. A universally acceptable selection criterion that takes the genotype $\mathrm{x}$ environment interaction into consideration does not exist. Whenever an interaction is significant, the use of main effects (e. $g$, means overall genotype across environments) is questionable (Kang and Magari, 1995). Abd El Bary (1999) found that locations and genotypes mean squares were highly significant for seed cotton per boll and lint percentage, seed index and lint index. El Oraby (1998) and El Ameer (1999) evaluated some Egyptian cotton genotypes under different environments. They reported that the mean squares of genotypes with respect to seed cotton per boll, seed index and lint percentage indices differed significantly. Baker (2001) evaluated some cotton genotypes under different environments. He found significant variations due to environments and genotypes with respect to yield (seed and lint). Idris (2002) evaluated some Egyptian cotton cultivars under different locations. He found that both the first analysis (locations, cultivars and the interaction between them) and the second analysis (environments, cultivars and the interaction between them) mean squares were significant with respect to seed and lint yield, seed cotton per boll, seed index and lint percentage. Mohamed et al. (2003) evaluated twenty-four cotton genotypes at six locations in Upper Egypt using the randomized complete block design. They found that genotypes $\mathrm{x}$ locations mean squares were highly significant for yields (seed and lint), seed cotton per boll and indices (seed index, lint index and lint percentage). The results showed that ((G83 x G80) $x$ G89)) was a promising cross due to its high performance for yield components and fiber quality. Hassan et al. (2012) evaluated some Egyptian cotton genotypes in different environments. They found that environments and genotype mean squares were highly significant for yields (seed and lint), seed cotton per boll, indices (seed index, lint index and lint percentage) and for fiber properties (fiber length, and micronaire reading).

Researchers need a statistical measure to Evaluate genotypes from environments and its 
components (locations and seasons). Thus, the objective of this study was to measure the response of some genotypes to different environments and their classification in two components (locations and seasons).

\section{MATERIALS AND METHODS}

Four long - staple Egyptian cotton (Gossypium barbadense L.) genotypes were evaluated in six environments in Upper Egypt. Environments consisted of three seasons (2009, 2010 and 2011) x two locations (Beni Souf and Minya) in Upper Egypt. Two of the genotypes were cultivars, viz. G.80 and G90. The other genotypes were hybrids (Bulk families) viz., (G83 x (G75 x 5844)) x G80 and G90 x Australian. A randomized complete block design with four replications was used. Two samples were obtained from each plot. Planting was during the last week of March. All agricultural practices were done as recommended.

Genotypes were evaluated for yields (seed and lint (kentar / feddan)), boll components (dry weight $(\mathrm{g})$, seed cotton $(\mathrm{g})$ and number of seeds), indices (harvest index per boll, seed index (g), lint index ( $\mathrm{g}$ ) and lint percentage) and fiber properties (length $(\mathrm{mm})$, micronaire and pressely index).

\subsection{Statistical analysis}

\subsubsection{Analysis of individual environments}

The analysis of the randomized complete block design was carried out for the data of individual environments to estimate the variance among genotypes in individual environments. Statistical analysis of randomized complete block design followed Little and Hills (1978) and Roger (1994).

\subsubsection{Analysis of combined environments}

Homogeneity test of variances (Bartlett test) was applied according to the procedures reported by Bailey (1994) before starting the analysis of combined. The combined randomized complete block design was carried out with the data of the six environments to estimate the environmental effects on genotypes (Table 1). Statistical analysis

Table(1):Analysis of variance of environmental effect on genotypes

\begin{tabular}{|c|c|}
\hline Source of variation & df \\
\hline Environments & $(\mathrm{e}-\mathbf{1})$ \\
Replications / Environments & e (r-1) \\
Genotypes & $(\mathrm{g}-1)$ \\
Genotypes x environments & $(\mathrm{g}-\mathbf{1})(\mathrm{e}-\mathbf{1})$ \\
Experimental error & $\mathrm{e}(\mathrm{r}-\mathbf{1})(\mathrm{g}-\mathbf{1})$ \\
\hline Total & $\mathrm{r}$ g e - 1 \\
\hline
\end{tabular}

followed to Gomez and Gomez (1984). Treatment means were compared by the least significant difference (L.S.D.) test as given by Steel and Torrie (1980). All comparisons were done at 0.05 level of significance.

\subsubsection{Classification of environments}

The data in (Table 2) show the classification of environmental effects on individual genotypes. Statistical analysis of classification of environmental effect on individual genotypes (Table 3) was followed Fowler et al. (1998). The means were compared by Tukey test as given by the same author. All comparisons were done at 0.05 level of significance.

\section{RESULTS AND DISCUSSION}

\subsection{Analysis of individual environments}

The analysis of variances in individual environments with respect to yield, boll components, indices and fiber properties revealed the presence of significant differences among the genotypes (Table 4). Non significant differences among the genotypes were noticed for yields (seed and lint) in six environments except one and two environments for seed cotton yield and lint cotton yield, respectively.

The results of boll components showed that significant variations due to genotypes were observed in five environments for dry weight per boll and number of seeds per boll. In contrast, the differences were insignificant for seed cotton per boll in six environments.

Significant variation due to genotypes was detected for indices (harvest index, seed index, lint index and lint percentage) in six environments except two environments with respect to harvest index and one environment for both seed index and lint percentage.

The analysis of variance of fiber properties revealed the presence of significant differences among genotypes for micronaire reading, fiber length and pressely index in four, three and two environments, respectively.

\subsection{Analysis of combined environments}

Homogeneity of variance test (Bartlett test) was not significant for boll components (dry weight per boll, seed cotton peer boll and number of seeds per boll), indices (harvest index, seed index, lint index and lint percentage) and fiber properties (fiber length, micronaire reading and pressely index). In contrast, Bartlett test was significant for yields (seed and lint). The analysis of variance showed significant variation due to environments, genotypes and their interaction (Table 5). 
Table (2): Two - way ANOVA of classification of the environmental effect on individual genotypes

\begin{tabular}{|c|c|c|c|c|}
\hline & \multicolumn{3}{|c|}{ Seasons } \\
\hline & & 2009 & 2010 & 2011 \\
\hline \multirow[t]{2}{*}{ Locations } & $\left(\mathbf{L}_{1}\right)$ & \begin{tabular}{l}
\multicolumn{1}{c}{ Environment (1) } \\
Plot size $=52 \mathrm{~m}^{2}$ \\
10 rows x $8 \mathrm{~m} \mathrm{x} 0.65 \mathrm{~m}$ \\
Number of plots $=4$ \\
Number of samples $=8$ \\
Sample size $=50$ bolls
\end{tabular} & $\begin{array}{l}\quad \text { Environment }(2) \\
\text { Plot size }=62.4 \mathrm{~m}^{2} \\
12 \text { rows } \times 8 \mathrm{~m} \mathrm{x} 0.65 \mathrm{~m} \\
\text { Number of plots }=4 \\
\text { Number of samples }=8 \\
\text { Sample size }=50 \text { bolls }\end{array}$ & $\begin{array}{l}\quad \text { Environment (3) } \\
\text { Plot size }=52 \mathrm{~m}^{2} \\
10 \text { rows x } 8 \mathrm{~m} \mathrm{x} 0.65 \mathrm{~m} \\
\text { Number of plots }=4 \\
\text { Number of samples }=8 \\
\text { Sample size }=50 \text { bolls }\end{array}$ \\
\hline & $\left(\mathbf{L}_{2}\right)$ & \begin{tabular}{l}
\multicolumn{1}{c}{ Environment $(\mathbf{4})$} \\
Plot size $=52 \mathrm{~m}^{2}$ \\
20 rows $\times 4 \mathrm{~m} \times 0.65 \mathrm{~m}$ \\
Number of plots $=4$ \\
Number of samples $=8$ \\
Sample size $=50$ bolls
\end{tabular} & $\begin{array}{l}\quad \text { Environment (5) } \\
\text { Plot size }=62.4 \mathrm{~m}^{2} \\
24 \text { rows x } 4 \mathrm{~m} \mathrm{x} 0.65 \mathrm{~m} \\
\text { Number of plots }=4 \\
\text { Number of samples }=8 \\
\text { Sample size }=50 \text { bolls }\end{array}$ & $\begin{array}{l}\quad \text { Environment }(6) \\
\text { Plot size }=52 \mathrm{~m}^{2} \\
20 \text { rows } \times 4 \mathrm{~m} \times 0.65 \mathrm{~m} \\
\text { Number of plots }=4 \\
\text { Number of samples }=8 \\
\text { Sample size }=50 \text { bolls }\end{array}$ \\
\hline
\end{tabular}

Significant differences among environments were observed for boll components, indices and fiber properties except pressely index indicating that these traits were affected by environments. Significant variation due to the genotypes was recorded for indices, fiber properties and boll components except for seed cotton per boll.

The results of boll components exhibited that both G80 and G90 x Australian had the highest value for dry weight per boll and number of seeds per boll, respectively. They significantly surpassed all other genotypes.

The results of indices revealed that G80 was the best genotype with respect to seed and lint, occupying the first rank and significantly exceeded all other genotypes. G90 did not differ significantly from G90 x Australian for harvest index, significantly surpassed all other genotypes. In contrast, non-significant variations among genotypes were recorded for lint percentage except for G90 (Table 6).

The results of fiber properties showed that G80 was the best genotype for fiber length, it did not significantly differ from (G83 x (G75 x 5844)) $\mathrm{x}$ G80 but significantly surpassed all other genotypes. G90 x Australian gave the highest value with respect to micronaire reading, it significantly exceeded other genotypes except for G80. On the other hand, non-significant variations among genotypes were recorded for pressely index except for G90 x Australian (Table 6).

A significant interaction between genotypes $\mathrm{x}$ environments was observed for boll components (dry weight per boll, seed cotton per boll and number of seeds per boll), indices (seed index and lint percentage) and fiber properties (fiber length and micronair reading) Table 5.

\subsection{Analysis of classification of environments}

The results in Table (7) show the analysis of variance of classification of environmental effect on individual genotypes with respect to yield, boll components, indices and fiber properties. The data in Table (8) show the means of yields (seed and lint), boll components (dry weight per boll, seed cotton per boll and number of seeds per boll), indices (harvest index, seed index, lint index and lint percentage) and fiber properties (fiber length, micronaire reading).

The results of yields (seed and lint) reveal that genotypes were more strongly influenced by different environments and components (locations and seasons) due to the variance of genotypes were significant.

The results of boll components show that G90 x Australian was the best genotype (more stable) for three boll components because the variance of this genotype was non-significant under different environments and components (locations and seasons).

(G83 x (G75 x 5844)) x G80 was more stable for number of seeds per boll due to the nonsignificant variance of this genotype under different environments and locations. On the other hand, the same genotype was not affected by seasons with respect to dry weight per boll and seed cotton per boll since its variance was nonsignificant.

G90 was the best genotype grown under different locations with respect to three boll components due to the non-significant variance of this genotype.

G80 was not affected by seasons and locations with respect to seed cotton per boll and number of seeds per boll, respectively due to the nonsignificant variance of this genotype.

The results of indices exhibited different response of genotypes grown under different environments and environmental components (locations and seasons)Genotypes were more strongly influenced by different environment and components(locations and seasons with respect to seed index and lint percentage due to the variance of genotypes were significant expect the variance of G90 x Australian (seasons)for lent percentage. 
Table (3): Analysis of variance of the classification of environmental effects on individual genotypes.

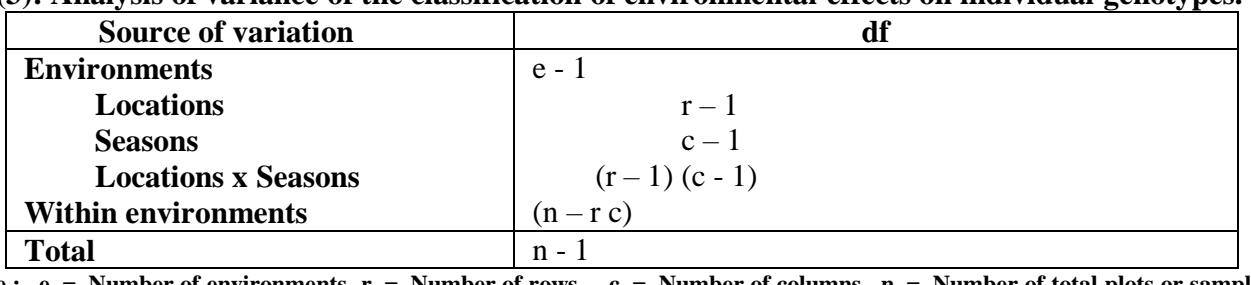

Where $: e=$ Number of environments $r=$ Number of rows $\quad c=$ Number of columns $n=$ Number of total plots or samples

Table (4): Mean squares of traits for individual environments.

\begin{tabular}{|c|c|c|c|c|c|c|c|}
\hline \multicolumn{8}{|c|}{ Seed cotton yield (k/fed.) } \\
\hline Source of variation & df & E (1) & E (2) & E (3) & E (4) & $\mathbf{E}(5)$ & $\mathbf{E}(6)$ \\
\hline Replications & 3 & $0.818^{*}$ & 2.54 & 1.69 & $2.91 *$ & 1.08 & 0.516 \\
\hline Genotypes & 3 & 0.447 & 0.243 & 2.21 & $3.07 *$ & 0.940 & 6.45 \\
\hline Experimental error & 9 & 0.167 & 0.913 & 1.81 & 0.472 & 1.55 & 3.77 \\
\hline Total & 15 & & & & & & \\
\hline \multicolumn{8}{|c|}{ Lint cotton yield (k/fed.) } \\
\hline Source of variation & df & E (1) & E (2) & E (3) & E (4) & E (5) & $\mathbf{E}(6)$ \\
\hline Replications & 3 & $1.29 *$ & 3.76 & 2.53 & $4.60 *$ & 1.85 & 0.998 \\
\hline Genotypes & 3 & $1.36 *$ & 0.564 & 4.74 & $4.64 *$ & 1.64 & 11.87 \\
\hline Experimental error & 9 & 0.237 & 1.78 & 2.89 & 0.810 & 3.69 & 6.22 \\
\hline Total & 15 & & & & & & \\
\hline \multicolumn{8}{|c|}{ Dry weight per boll (g) } \\
\hline Source of variation & df & E (1) & E (2) & E (3) & E (4) & $\mathbf{E}(5)$ & E (6) \\
\hline Replications & 7 & 0.005 & 0.017 & 0.007 & 0.006 & 0.005 & 0.011 \\
\hline Genotypes & 3 & $0.096 * *$ & $0.093 * *$ & 0.013 & $0.109 * *$ & $0.071 *$ & $0.098 * *$ \\
\hline Experimental error & 21 & 0.006 & 0.009 & 0.007 & 0.005 & 0.021 & 0.014 \\
\hline Total & 31 & & & & & & \\
\hline \multicolumn{8}{|c|}{ Seed cotton per boll $(\mathrm{g})$} \\
\hline Source of variation & df & E (1) & E (2) & E (3) & E (4) & E (5) & E (6) \\
\hline Replications & 7 & 0.023 & 0.046 & 0.044 & 0.023 & 0.035 & 0.126 \\
\hline Genotypes & 3 & 0.052 & 0.136 & 0.172 & 0.149 & 0.063 & 0.122 \\
\hline Experimental error & 21 & 0.049 & 0.078 & 0.057 & 0.065 & 0.040 & 0.056 \\
\hline Total & 31 & & & & & & \\
\hline \multicolumn{8}{|c|}{ Number of seeds per boll } \\
\hline Source of variation & df & E (1) & E (2) & E (3) & E (4) & $\mathbf{E}(5)$ & E (6) \\
\hline Replications & 7 & 1.53 & 2.08 & 2.32 & 1.39 & 2.47 & 3.40 \\
\hline Genotypes & 3 & $31.58 * *$ & $11.37 *$ & $23.36 * *$ & $6.87 *$ & 1.87 & $12.31 * *$ \\
\hline Experimental error & 21 & 2.24 & 3.57 & 1.90 & 2.00 & 1.81 & 2.05 \\
\hline Total & 31 & & & & & & \\
\hline \multicolumn{8}{|c|}{ Harvest index per boll (\%) } \\
\hline Source of variation & df & E (1) & $\mathbf{E}(2)$ & E (3) & E (4) & $\mathbf{E}(5)$ & E (6) \\
\hline Replications & 7 & 0.027 & 0.057 & 0.026 & 0.039 & 0.011 & 0.139 \\
\hline Genotypes & 3 & $0.540 * *$ & $0.385 * *$ & 0.224 & $0.226^{*}$ & 0.209 & $0.513 * *$ \\
\hline Experimental error & 21 & 0.042 & 0.070 & 0.084 & 0.063 & 0.074 & 0.060 \\
\hline Total & 31 & & & & & & \\
\hline \multicolumn{8}{|c|}{ Seed index $(\mathrm{g})$} \\
\hline Source of variation & df & $\mathbf{E}(\mathbf{1})$ & E (2) & E (3) & E (4) & E (5) & $E(6)$ \\
\hline Replications & 7 & 0.331 & 0.223 & 0.175 & 0.059 & 0.609 & $0.774 *$ \\
\hline Genotypes & 3 & $5.39 * *$ & $1.30 * *$ & $3.54 * *$ & $5.53 * *$ & 0.950 & $3.12 * *$ \\
\hline Experimental error & 21 & 0.163 & 0.193 & 0.397 & 0.194 & 0.513 & 0.277 \\
\hline Total & 31 & & & & & & \\
\hline \multicolumn{8}{|c|}{ Lint index (g) } \\
\hline Source of variation & df & E (1) & E (2) & E (3) & E (4) & $\mathbf{E}(5)$ & $\mathbf{E}(6)$ \\
\hline Replications & 7 & 0.165 & 0.111 & 0.187 & 0.133 & 0.164 & 0.301 \\
\hline Genotypes & 3 & $2.80 * *$ & $1.05^{* *}$ & $1.60 * *$ & $1.83 * *$ & $1.02 *$ & $0.788 * *$ \\
\hline Experimental error & 21 & 0.070 & 0.092 & 0.172 & 0.072 & 0.228 & 0.139 \\
\hline Total & 31 & & & & & & \\
\hline \multicolumn{8}{|c|}{ Lint percentage } \\
\hline Source of variation & df & E (1) & E (2) & E (3) & E (4) & E (5) & $\mathbf{E}(6)$ \\
\hline Replications & 7 & 1.00 & 0.416 & 0.652 & 1.43 & 0.904 & 0.593 \\
\hline Genotypes & 3 & $10.66 * *$ & $5.43 * *$ & 1.69 & $5.53 * *$ & $3.68 *$ & $4.46^{* *}$ \\
\hline Experimental error & 21 & 0.552 & 0.420 & 0.646 & 0.770 & 0.765 & 0.621 \\
\hline Total & 31 & & & & & & \\
\hline
\end{tabular}


Table (4) Cont.

\begin{tabular}{|c|c|c|c|c|c|c|c|}
\hline \multicolumn{8}{|c|}{ Fiber length (mm) } \\
\hline Source of variation & df & E (1) & E (2) & E (3) & E (4) & E (5) & E (6) \\
\hline Replications & 7 & 0.206 & 0.062 & 0.514 & 0.523 & 0.364 & 0.758 \\
\hline Genotypes & 3 & $1.26 *$ & $1.04 *$ & $2.05 *$ & 0.614 & 0.512 & 1.57 \\
\hline Experimental error & 21 & 0.360 & 0.334 & 0.476 & 0.271 & 0.386 & 0.584 \\
\hline Total & 31 & & & & & & \\
\hline \multicolumn{8}{|c|}{ Micronaire reading } \\
\hline Source of variation & df & E (1) & $E(2)$ & E (3) & E (4) & $E(5)$ & E (6) \\
\hline Replications & 7 & 0.020 & 0.009 & 0.065 & 0.024 & 0.047 & 0.036 \\
\hline Genotypes & 3 & $0.129 * *$ & 0.015 & $0.145^{*}$ & $0.172 *$ & 0.096 & 0.194* \\
\hline Experimental error & 21 & 0.021 & 0.031 & 0.031 & 0.035 & $\mathbf{0 . 0 3 7}$ & 0.058 \\
\hline Total & 31 & & & & & & \\
\hline \multicolumn{8}{|c|}{ Pressely index } \\
\hline Source of variation & df & E (1) & E (2) & E (3) & E (4) & E (5) & $\mathbf{E}(6)$ \\
\hline Replications & 7 & 0.517 & $2.22 * *$ & 0.123 & 0.183 & 0.734 & 0.108 \\
\hline Genotypes & 3 & 0.064 & 0.057 & 0.314 & 0.514 & $2.32 *$ & $0.608 *$ \\
\hline Experimental error & 21 & 0.363 & 0.521 & 0.170 & 0.248 & 0.598 & 0.133 \\
\hline Total & 31 & & & & & & \\
\hline
\end{tabular}

* , ** Significant at 0.05 and 0.01 levels, respectively.E = Environment

Table (5): Mean squares of traits for combined analysis.

\begin{tabular}{|c|c|c|c|}
\hline Source of variation & df & Seed cotton yield (k/fed.) & Lint cotton yield (k/fed.) \\
\hline Environments (E) & 5 & -- & -- \\
\hline Replications / (E) & 18 & -- & -- \\
\hline Genotypes (G) & 3 & -- & -- \\
\hline GXE & 15 & -- & -- \\
\hline Experimental error & 54 & \multirow[t]{2}{*}{--} & \multirow[t]{2}{*}{--} \\
\hline Total & 95 & & \\
\hline Source of variation & df & Dry weight per boll (g) & Seed cotton per boll $(\mathrm{g})$ \\
\hline Environments (E) & 5 & $0.105 * *$ & $0.445 * *$ \\
\hline Replications / (E) & 42 & 0.008 & 0.050 \\
\hline Genotypes (G) & 3 & $0.355 * *$ & 0.027 \\
\hline $\mathbf{G} \times \mathbf{E}$ & 15 & $0.025 * *$ & $0.133 * *$ \\
\hline Experimental error & 126 & \multirow[t]{2}{*}{0.010} & \multirow[t]{2}{*}{0.058} \\
\hline Total & 191 & & \\
\hline Source of variation & df & Number of seeds per boll & Harvest index (\%) \\
\hline Environments (E) & 5 & 23.07** & $0.247 * *$ \\
\hline Replications / (E) & 42 & 2.20 & 0.050 \\
\hline Genotypes (G) & 3 & $57.54 * *$ & $1.91 * *$ \\
\hline $\mathbf{G} \times \mathbf{E}$ & 15 & $5.96 * *$ & 0.037 \\
\hline Experimental error & 126 & \multirow[t]{2}{*}{2.26} & \multirow{2}{*}{0.066} \\
\hline Total & 191 & & \\
\hline Source of variation & df & Seed index (g) & Lint index (g) \\
\hline Environments (E) & 5 & $14.20 * *$ & $2.29 * *$ \\
\hline Replications / (E) & 42 & 0.362 & 0.177 \\
\hline Genotypes (G) & 3 & $16.24 * *$ & $8.02 * *$ \\
\hline$G \times E$ & 15 & $0.718 * *$ & 0.213 \\
\hline Experimental error & 126 & \multirow[t]{2}{*}{0.290} & \multirow[t]{2}{*}{0.129} \\
\hline Total & 191 & & \\
\hline Source of variation & df & Lint percentage & Fiber length (mm) \\
\hline Environments (E) & 5 & $31.70 * *$ & $52.78 * *$ \\
\hline Replications / (E) & 42 & 0.833 & 0.404 \\
\hline Genotypes (G) & 3 & $22.67 * *$ & $1.11 *$ \\
\hline$G \times E$ & 15 & $1.76 * *$ & $1.19 * *$ \\
\hline Experimental error & 126 & \multirow[t]{2}{*}{0.629} & \multirow[t]{2}{*}{0.402} \\
\hline Total & 191 & & \\
\hline Source of variation & df & Micronaire reading & Pressely index \\
\hline Environments (E) & 5 & $1.34 * *$ & 1.32 \\
\hline Replications / (E) & 42 & 0.033 & 0.647 \\
\hline Genotypes (G) & 3 & $0.368 * *$ & $1.15 *$ \\
\hline $\mathbf{G} \times \mathbf{E}$ & 15 & $0.077 *$ & 0.545 \\
\hline Experimental error & 126 & \multirow[t]{2}{*}{0.036} & \multirow[t]{2}{*}{0.339} \\
\hline Total & 191 & & \\
\hline
\end{tabular}


Table (6): Means of boll components, indices and fiber properties for combined analysis.

\begin{tabular}{|c|c|c|}
\hline Genotypes & Seed cotton yield (k/fed.) & Lint cotton yield (k/fed.) \\
\hline $\begin{array}{c}\text { G80 } \\
\text { G90 } \\
(\text { G83 x }(\text { G75 x 5844) }) \times \text { G80 } \\
\text { G90 x Australian } \\
\text { L. S. D. }\end{array}$ & $\begin{array}{l}-- \\
-- \\
-- \\
--\end{array}$ & $\begin{array}{l}-- \\
-- \\
-- \\
--\end{array}$ \\
\hline Genotypes & Dry weight per boll (g) & Seed cotton per boll (g) \\
\hline $\begin{array}{c}\text { G80 } \\
\text { G90 } \\
(\text { G83 x }(\text { G75 x 5844)) x G80 } \\
\text { G90 x Australian } \\
\text { L. S. D. } \\
\end{array}$ & $\begin{array}{l}1.12 \\
0.93 \\
1.00 \\
0.95\end{array}$ & $\begin{array}{l}2.58 \\
2.53 \\
2.56 \\
2.58\end{array}$ \\
\hline Genotypes & Number of seeds per boll & Harvest index (\%) \\
\hline $\begin{array}{c}\text { G80 } \\
\text { G90 } \\
(\text { G83 x }(\text { G75 x 5844) }) \times \text { x G80 } \\
\text { G90 x Australian } \\
\text { L. S. D. } \\
\end{array}$ & $\begin{array}{l}15.38 \\
16.08 \\
16.15 \\
17.95\end{array}$ & $\begin{array}{l}2.31 \\
2.74 \\
2.56 \\
2.73\end{array}$ \\
\hline Genotypes & Seed index (g) & Lint index $(\mathrm{g})$ \\
\hline $\begin{array}{c}\text { G80 } \\
\text { G90 } \\
(\text { G83 x }(\text { G75 x 5844) }) \times \text { x80 } \\
\text { G90 x Australian } \\
\text { L. S. D. } \\
\end{array}$ & $\begin{array}{l}9.95 \\
9.58 \\
9.45 \\
8.58\end{array}$ & $\begin{array}{l}6.85 \\
6.21 \\
6.46 \\
5.88\end{array}$ \\
\hline Genotypes & Lint percentage & Fiber length $(\mathrm{mm})$ \\
\hline $\begin{array}{c}\text { G80 } \\
\text { G90 } \\
(\text { G83 x }(\text { G75 x 5844) }) \text { x G80 } \\
\text { G90 x Australian } \\
\text { L. S. D. }\end{array}$ & $\begin{array}{l}40.81 \\
39.34 \\
40.62 \\
40.69\end{array}$ & $\begin{array}{l}32.56 \\
32.25 \\
32.32 \\
32.23\end{array}$ \\
\hline Genotypes & Micronaire reading & Pressely index \\
\hline $\begin{array}{c}\text { G80 } \\
\text { G90 } \\
(\text { G83 x }(\text { G75 x 5844) }) \times \text { x G80 } \\
\text { G90 x Australian } \\
\text { L. S. D. }\end{array}$ & $\begin{array}{l}4.45 \\
4.28 \\
4.35 \\
4.47\end{array}$ & \begin{tabular}{c|}
10.17 \\
10.25 \\
10.02 \\
9.90
\end{tabular} \\
\hline
\end{tabular}

ns : Not significant at .05 level.

-- Not combined analysis due to Bartlett test was significant.

In contrast, the genotypes were more stable for harvest index due to the variances of genotypes was non-significant under different environments and components (locations and seasons).

The results of lint index showed that the genotypes were more strongly influenced by different environments and seasons since the variance of genotypes was significant. The genotypes were not affected by locations with respect to the same trait since the variance of these genotypes were non-significant except G90 x Australian.

The results of fiber properties revealed that the genotypes were more strongly influenced by different environments and seasons with respect to fiber length and micronaire reading since the variance of genotypes was significant. In contrast, the genotypes were not affected by environments and seasons with respect to pressely index except (G83 x (G75 x 5844)) x G80 where the variance of the genotypes was non-significant.

On the other hand, the genotypes were not affected by locations with respect to the three characters except G80 for fiber length and G90 x Australian for pressely index since to the variance of genotypes was non-significant.

G80 and G90 were the best genotypes (more stable) with respect to pressely index because they were not affected by environments and environmental components (locations and seasons) since the variances of the two genotypes was non-significant. 
Table (7): Mean squares of classification environmental effect on individual genotypes. Seed cotton yield (k/fed.)

\begin{tabular}{|c|c|c|c|c|c|}
\hline Source of variation & dff & G80 & G90 & $V_{1}$ & $\mathbf{V}_{2}$ \\
\hline Environments & 5 & \multirow{6}{*}{$\begin{array}{c}16.99 * * \\
50.43 * * \\
16.43 * * \\
0.83 \\
1.45\end{array}$} & \multirow{6}{*}{$\begin{array}{l}\text { 32.62** } \\
\text { 93.46** } \\
34.17 * * \\
0.66 \\
0.86\end{array}$} & \multirow{6}{*}{$\begin{array}{c}26.79 * * \\
95.32 * * \\
19.12 * * \\
1.84\end{array}$} & \multirow{6}{*}{$\begin{array}{c}41.59 * * \\
76.86 * * \\
64.80 * * \\
1.78\end{array}$} \\
\hline Locations & 1 & & & & \\
\hline Seasons & 2 & & & & \\
\hline Locations x Seasons & 2 & & & & \\
\hline Within environments & 18 & & & & \\
\hline Total & 23 & & & & \\
\hline
\end{tabular}

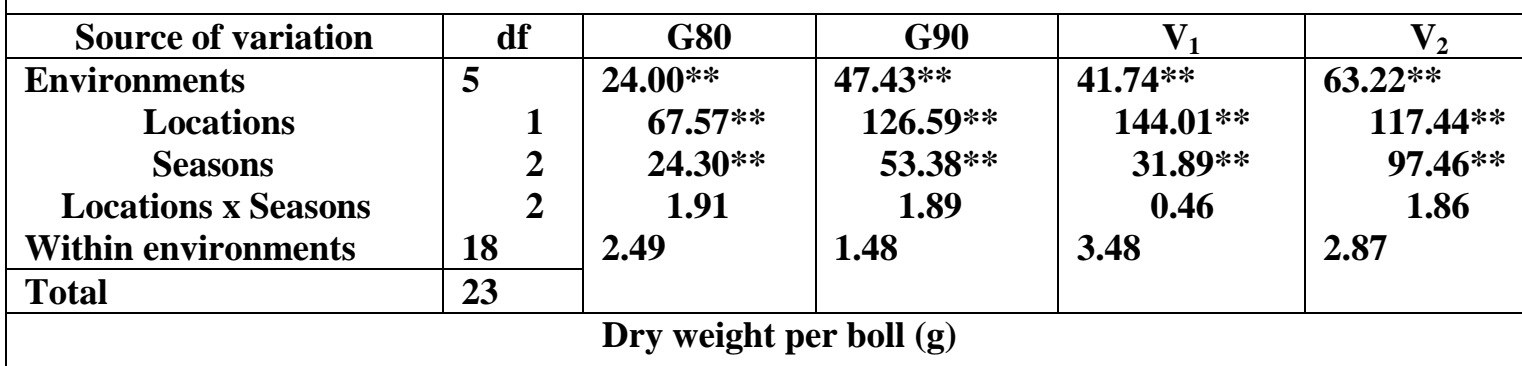

\begin{tabular}{|c|c|c|c|c|c|}
\hline Source of variation & dff & G80 & G90 & $V_{1}$ & $V_{2}$ \\
\hline Environments & 5 & $0.087 * *$ & $0.064 * *$ & $0.020 * *$ & 0.007 \\
\hline Locations & 1 & $0.134 * *$ & 0.009 & $0.057 * *$ & 0.005 \\
\hline Seasons & 2 & $0.081 *$ & $0.125 * *$ & 0.011 & 0.010 \\
\hline Locations x Seasons & 2 & $0.069 *$ & $0.031 *$ & 0.011 & 0.006 \\
\hline Within environments & 42 & 0.016 & 0.008 & 0.004 & 0.011 \\
\hline Total & 47 & & & & \\
\hline
\end{tabular}

\begin{tabular}{|c|c|c|c|c|c|}
\hline Source of variation & df & G80 & G90 & $V_{1}$ & $\mathbf{V}_{2}$ \\
\hline Environments & 5 & $0.336 * *$ & $0.208 * *$ & $0.205 * *$ & 0.097 \\
\hline Locations & $\mathbf{1}$ & $0.788 * *$ & 0.004 & $0.391 * *$ & 0.103 \\
\hline Seasons & 2 & 0.180 & $0.278 * *$ & 0.020 & 0.043 \\
\hline Locations x Seasons & 2 & $0.266 *$ & $0.241 *$ & $0.296 * *$ & 0.148 \\
\hline Within environments & 42 & 0.073 & 0.049 & 0.044 & 0.056 \\
\hline Total & 47 & & & & \\
\hline
\end{tabular}

\begin{tabular}{|c|c|c|c|c|c|}
\hline Source of variation & df & G80 & G90 & $\mathbf{V}_{1}$ & $\mathbf{V}_{2}$ \\
\hline $\begin{array}{c}\text { Environments } \\
\text { Locations } \\
\text { Seasons } \\
\text { Locations x Seasons } \\
\text { Within environments }\end{array}$ & $\begin{array}{ll}5 & \\
& 1 \\
& 2 \\
& 2 \\
42 & \end{array}$ & \multirow[t]{2}{*}{$\begin{array}{c}14.75^{* *} \\
5.95 \\
28.63 * * \\
5.28 \\
2.09\end{array}$} & \multirow[t]{2}{*}{$\begin{array}{c}15.58 * * \\
1.33 \\
36.82 * * \\
1.45 \\
1.74\end{array}$} & \multirow[t]{2}{*}{$\begin{array}{l}4.07 \\
1.62 \\
7.49 * \\
1.87 \\
2.16\end{array}$} & \multirow[t]{2}{*}{$\begin{array}{r}6.55 \\
11.52 \\
3.58 \\
7.04 \\
3.00\end{array}$} \\
\hline Total & 47 & & & & \\
\hline \multicolumn{6}{|c|}{ Harvest index per boll (\%) } \\
\hline Source of variation & df & G80 & G90 & $\mathbf{V}_{1}$ & $\mathbf{V}_{2}$ \\
\hline $\begin{array}{c}\text { Environments } \\
\text { Locations } \\
\text { Seasons } \\
\text { Locations x Seasons } \\
\text { Within environments }\end{array}$ & $42 \begin{array}{r}1 \\
2 \\
2\end{array}$ & \multirow[t]{2}{*}{$\begin{array}{l}0.100 \\
0.005 \\
0.093 \\
0.155 \\
0.062\end{array}$} & \multirow[t]{2}{*}{$\begin{array}{r}0.096 \\
0.066 \\
0.207 \\
0.001 \\
0.064\end{array}$} & \multirow[t]{2}{*}{$\begin{array}{c}0.084 \\
0.010 \\
0.042 \\
0.167 * \\
0.043\end{array}$} & \multirow[t]{2}{*}{$\begin{array}{r}0.078 \\
0.030 \\
0.113 \\
0.068 \\
0.076\end{array}$} \\
\hline Total & 47 & & & & \\
\hline
\end{tabular}


Table (7): Cont.

\begin{tabular}{|c|c|c|c|c|c|}
\hline \multicolumn{6}{|c|}{ Seed index (g) } \\
\hline Source of variation & df & G80 & G90 & $\mathbf{V}_{1}$ & $\mathbf{V}_{2}$ \\
\hline Environments & 5 & $5.42 * *$ & $3.40 * *$ & $3.67 * *$ & $3.87 * *$ \\
\hline Locations & 1 & 8.34** & $2.81 * *$ & $4.64 * *$ & $9.77 * *$ \\
\hline Seasons & 2 & $6.52 * *$ & 3.79** & $2.19 * *$ & $2.51 * *$ \\
\hline Locations x Seasons & 2 & $2.85 * *$ & $3.31 * *$ & $4.65 * *$ & $2.29 * *$ \\
\hline Within environments & 42 & 0.405 & 0.314 & 0.337 & 0.175 \\
\hline Total & 47 & & & & \\
\hline \multicolumn{6}{|c|}{ Lint index (g) } \\
\hline Source of variation & df & G80 & G90 & $\mathbf{V}_{1}$ & $\mathbf{V}_{2}$ \\
\hline Environments & 5 & $0.616 *$ & $0.731 * *$ & $0.747 * *$ & 0.839** \\
\hline Locations & 1 & $\mathbf{0 . 0 3 0}$ & 0.003 & 0.368 & $1.56 * *$ \\
\hline Seasons & 2 & $1.15 * *$ & $1.15 * *$ & 0.175 & $0.999 * *$ \\
\hline Locations x Seasons & 2 & 0.374 & $0.679 * *$ & $1.51 * *$ & 0.322 \\
\hline Within environments & 42 & 0.206 & 0.128 & 0.128 & 0.101 \\
\hline Total & 47 & & & & \\
\hline \multicolumn{6}{|c|}{ Lint percentage } \\
\hline Source of variation & df & G80 & G90 & $\mathbf{V}_{1}$ & $\mathbf{V}_{2}$ \\
\hline Environments & 5 & $14.75 * *$ & $8.69 * *$ & $6.82 * *$ & $6.72 * *$ \\
\hline Locations & 1 & $38.47 * *$ & $18.09 * *$ & $9.48 * *$ & $14.01 * *$ \\
\hline Seasons & 2 & $6.12 * *$ & $4.75 * *$ & $8.42 * *$ & 0.190 \\
\hline Locations x Seasons & 2 & $11.53 * *$ & $7.93 * *$ & $3.87 *$ & $9.59 * *$ \\
\hline Within environments & 42 & 0.617 & 0.460 & 0.953 & 0.689 \\
\hline Total & 47 & & & & \\
\hline \multicolumn{6}{|c|}{ Fiber length (mm) } \\
\hline Source of variation & df & G80 & G90 & $\overline{V_{1}}$ & $\mathrm{~V}_{2}$ \\
\hline Environments & 5 & $10.66 * *$ & $22.65 * *$ & $14.10 * *$ & $8.04 * *$ \\
\hline Locations & 1 & $3.52 * *$ & 0.060 & 0.075 & 0.054 \\
\hline Seasons & 2 & $23.22 * *$ & $53.74 * *$ & $37.31 * *$ & $19.74 * *$ \\
\hline Locations x Seasons & 2 & $1.67 *$ & $2.85 * *$ & 0.149 & 0.339 \\
\hline Within environments & 42 & 0.337 & 0.386 & 0.343 & 0.543 \\
\hline Total & 47 & & & & \\
\hline \multicolumn{6}{|c|}{ Micronaire reading } \\
\hline Source of variation & df & G80 & G90 & $\mathbf{V}_{1}$ & $\mathbf{V}_{2}$ \\
\hline Environments & 5 & $0.256 * *$ & $0.266 * *$ & $0.483 * *$ & $0.565 * *$ \\
\hline Locations & 1 & 0.047 & 0.005 & 0.010 & 0.041 \\
\hline Seasons & 2 & $0.334 * *$ & $0.654 * *$ & $1.03 * *$ & $1.34 * *$ \\
\hline Locations x Seasons & 2 & $0.283 * *$ & 0.009 & $0.183 * *$ & 0.051 \\
\hline Within environments & 42 & 0.037 & 0.034 & 0.030 & 0.039 \\
\hline Total & 47 & & & & \\
\hline \multicolumn{6}{|c|}{ Pressely index } \\
\hline Source of variation & df & G80 & G90 & $\mathbf{V}_{1}$ & $\mathbf{V}_{2}$ \\
\hline Environments & 5 & 0.309 & 0.325 & $1.14 *$ & 1.18 \\
\hline Locations & 1 & 0.001 & 0.060 & 0.002 & $3.58 * *$ \\
\hline Seasons & 2 & 0.239 & 0.295 & $2.31 *$ & 0.781 \\
\hline Locations x Seasons & 2 & 0.533 & 0.488 & 0.548 & 0.391 \\
\hline Within environments & 42 & 0.347 & 0.372 & 0.459 & 0.485 \\
\hline Total & 47 & & & & \\
\hline
\end{tabular}

,** Significant at 0.05 and 0.01 levels, respectively.

$\mathrm{V}_{1}=(\mathbf{G 8 3} \times(\mathbf{G 7 5} \times \mathbf{5 8 4 4})) \times \mathbf{G 8 0}$

$\mathrm{V}_{2}=\mathbf{G 9 0} \times$ Australian 
Table (8): Means of yield, boll components, indices and fiber properties for classification environments.

\begin{tabular}{|c|c|c|c|c|c|c|c|c|}
\hline \multirow[b]{2}{*}{ Environments } & \multicolumn{4}{|c|}{ Seed cotton yield (k/fed.) } & \multicolumn{4}{|c|}{ Lint cotton yield (k/fed.) } \\
\hline & G80 & G90 & $\mathbf{V}_{1}$ & $\mathbf{V}_{2}$ & G80 & G90 & $\mathbf{V}_{1}$ & $\mathbf{V}_{2}$ \\
\hline (1) & 7.01 & 6.42 & 7.04 & 6.47 & 9.27 & 8.03 & 9.16 & 8.51 \\
\hline (2) & 8.88 & 8.91 & 9.21 & 9.39 & 11.57 & 11.21 & 12.01 & 11.97 \\
\hline (3) & 10.18 & 9.97 & 10.39 & 11.63 & 12.74 & 12.34 & 13.15 & 14.82 \\
\hline (4) & 10.05 & 9.73 & 11.37 & 9.35 & 12.46 & 11.69 & 14.08 & 11.91 \\
\hline (5) & 12.34 & 13.33 & 13.10 & 13.40 & 15.98 & 16.82 & 17.38 & 17.29 \\
\hline (6) & 12.38 & 14.08 & 14.13 & 15.48 & 15.22 & 16.86 & 17.57 & 19.37 \\
\hline Tukey & 2.70 & 2.08 & 3.05 & 3.00 & 3.54 & 2.74 & 4.19 & 3.80 \\
\hline Environments & \multicolumn{4}{|c|}{ Dry weight per boll (g) } & \multicolumn{4}{|c|}{ Harvest index per boll $(\%)$} \\
\hline (1) & 1.08 & 0.81 & 0.95 & 0.93 & 2.25 & 2.85 & 2.44 & 2.65 \\
\hline (2) & 1.21 & 1.06 & 0.97 & 0.98 & 2.20 & 2.64 & 2.55 & 2.68 \\
\hline (3) & 0.93 & 0.88 & 0.98 & 0.91 & 2.45 & 2.83 & 2.70 & 2.78 \\
\hline (4) & 1.17 & 0.92 & 1.03 & 0.93 & 2.45 & 2.79 & 2.67 & 2.82 \\
\hline (5) & 1.18 & 1.00 & 1.09 & 0.98 & 2.24 & 2.57 & 2.49 & 2.60 \\
\hline (6) & 1.17 & 0.92 & 0.99 & 0.98 & 2.27 & 2.73 & 2.54 & 2.84 \\
\hline Tukey & 0.19 & 0.13 & 0.10 & -- & -- & -- & -- & -- \\
\hline Environments & \multicolumn{4}{|c|}{ Seed cotton per boll (g) } & \multicolumn{4}{|c|}{ Seed index $(\mathrm{g})$} \\
\hline (1) & 2.42 & 2.29 & 2.32 & 2.46 & 9.27 & 8.99 & 8.83 & 7.43 \\
\hline (2) & 2.65 & 2.79 & 2.47 & 2.63 & 9.15 & 9.19 & 8.92 & 8.31 \\
\hline (3) & 2.28 & 2.49 & 2.63 & 2.53 & 10.18 & 9.85 & 9.66 & 8.64 \\
\hline (4) & 2.87 & 2.56 & 2.73 & 2.63 & 11.05 & 10.47 & 10.67 & 9.14 \\
\hline (5) & 2.61 & 2.57 & 2.72 & 2.51 & 9.32 & 8.89 & 9.15 & 8.53 \\
\hline (6) & 2.63 & 2.49 & 2.51 & 2.76 & 10.73 & 10.11 & 9.45 & 9.42 \\
\hline Tukey & 0.40 & 0.32 & 0.31 & -- & 0.94 & $\mathbf{0 . 8 2}$ & 0.85 & 0.62 \\
\hline Environments & \multicolumn{4}{|c|}{ Number of seeds per boll } & \multicolumn{4}{|c|}{ Lint index (g) } \\
\hline (1) & 15.04 & 15.38 & 15.54 & 19.27 & 6.80 & 5.94 & 6.06 & 5.36 \\
\hline (2) & 16.93 & 18.33 & 16.33 & 18.89 & 6.47 & 6.02 & 6.24 & 5.62 \\
\hline (3) & 13.11 & 15.04 & 16.01 & 17.14 & 7.19 & 6.69 & 6.80 & 6.11 \\
\hline (4) & 15.85 & 15.21 & 15.62 & 17.33 & 7.10 & 6.37 & 6.82 & 6.02 \\
\hline (5) & 16.43 & 17.33 & 17.48 & 17.36 & 6.61 & 5.93 & 6.48 & 5.94 \\
\hline (6) & 14.90 & 15.21 & 15.89 & 17.67 & 6.90 & 6.31 & 6.34 & 6.21 \\
\hline Tukey & 2.12 & 1.94 & -- & -- & 0.67 & 0.53 & 0.53 & 0.47 \\
\hline Environments & \multicolumn{4}{|c|}{ Fiber length (mm) } & \multicolumn{4}{|c|}{ Lint percentage } \\
\hline (1) & 33.53 & 33.64 & 33.15 & 32.76 & 42.30 & 39.78 & 40.70 & 41.92 \\
\hline (2) & 33.65 & 33.06 & 33.30 & 32.80 & 41.43 & 39.62 & 41.18 & 40.34 \\
\hline (3) & 31.31 & 30.15 & 30.61 & 31.02 & 41.38 & 40.47 & 41.32 & 41.44 \\
\hline (4) & 32.24 & 32.70 & 32.89 & 32.70 & 39.10 & 37.72 & 38.99 & 39.70 \\
\hline (5) & 33.49 & 33.81 & 33.43 & 33.20 & 41.52 & 40.02 & 41.38 & 41.02 \\
\hline (6) & 31.14 & 30.13 & 30.51 & 30.89 & 39.12 & 38.44 & 40.16 & 39.73 \\
\hline Tukey & 0.85 & 0.91 & 0.86 & 1.08 & 1.15 & 1.00 & 1.44 & 1.22 \\
\hline Environments & \multicolumn{4}{|c|}{ Micronaire reading } & \multicolumn{4}{|c|}{ Pressely index } \\
\hline (1) & 4.44 & 4.18 & 4.16 & 4.25 & 10.30 & 10.35 & 10.21 & 10.42 \\
\hline (2) & 4.23 & 4.19 & 4.16 & 4.26 & 10.06 & 10.08 & 10.04 & 10.23 \\
\hline (3) & 4.78 & 4.51 & 4.74 & 4.81 & 10.15 & 10.21 & 9.81 & 9.88 \\
\hline (4) & 4.43 & 4.10 & 4.14 & 4.26 & 10.10 & 10.35 & 10.45 & 9.89 \\
\hline (5) & 4.40 & 4.20 & 4.39 & 4.45 & 10.48 & 10.53 & 10.20 & 9.36 \\
\hline (6) & 4.43 & 4.51 & 4.54 & 4.79 & 9.91 & 9.98 & 9.38 & 9.64 \\
\hline Tukey & 0.28 & 0.27 & 0.25 & 0.29 & -- & -- & $\mathbf{1 . 0 0}$ & -- \\
\hline
\end{tabular}

$\mathrm{V}_{1}=(\mathbf{G 8 3} \times(\mathbf{G 7 5} \times 5844)) \times$ G80 $\quad V_{2}=$ G90 x Australian $\quad--$ Not significant at .05 level.

\section{REFERENCES}

Abd El Bary A. M. (1999). Inheritance of quantitive traits of Egyptian cotton (Gossypium barbadense L.). M. Sc. Thesis, Mansoura Univ., Egypt.

Bailey N. T. (1994). Statistical Methods in Biology. Third Edit. Cambridge University Press, Cambridge, England.
Baker K. M. (2001). Comparative performance of Pima and Egyptian cotton under local environmental conditions. M.Sc. Thesis, Fac., of Agric., Cairo, Univ., Egypt.

El Ameer M. A. (1999). Genetical studies on types of gene effects on some crosses of Egyptian cotton (Gossypium barbadense L.). M. Sc. Thesis,Mansoura Univ., Egypt. 
El Oraby S.H. (1998). Studies on yield of some Egyptian cotton varieties under different environments. M.Sc. Thesis, Kafr El Sheikh, Tanta Univ., Egypt.

Hassan S. A., Badr S.S. and Hassan I.S. (2012). Study of phenotypic stability of some Egyptian cotton genotypes under different environments. Egypt. J. of Appl. Sci., (6) : $298-315$.

Fowler J., Cohen L. and Jarvis P. (1998). Practical statistics for field biology. Second Edit, John Wiley and Sons, New York, U.S.A.

Gomez K. A. and Gomez A. A. (1984). Statistical Procedures for Agricultural Research. John Wiley and Sons, New York, U.S.A.

Idris H. A. (2002). Models for evaluating Egyptian cotton responses to environmental variables. Ph.D. Thesis, Fac., of Agric., Cairo Univ., Egypt
Kang, M. S and Magari R. (1995). Stable a BASICA program for calculating stability and yield - stability statistics. Agron. J. 87 : $276-277$.

Little T.M. and Hills F. J.(1978). Agricultural Experimentation Design and Analysis. John Wiley and Sons, New York., U.S.A.

Mohamed S. A., El Adly H. H. and Eissa A. E. (2003). Evaluation of some Egyptian cotton genotypes under different environments. Egypt. J . Agric. Res., 81 (4) : 1797- 1816.

Roger G. P. (1994). Agricultural Field Experiments Design and Analysis. Marcel Dekker, Inc. New York, U.S.A.

Steel R. G. and Torrie J. H. (1980). Principles and Procedures of Statistics. Second Edit., McGraw. Hill. Book Co. New York, U.S.A. 